\title{
MR Elastography of the Abdomen: Experimental Protocols
}

\section{Suraj D. Serai and Meng Yin}

\begin{abstract}
Application of MRE for noninvasive evaluation of renal fibrosis has great potential for noninvasive assessment in patients with chronic kidney disease (CKD). CKD leads to severe complications, which require dialysis or kidney transplant and could even result in death. CKD in native kidneys and interstitial fibrosis in allograft kidneys are the two major kidney fibrotic pathologies where MRE may be clinically useful. Both these conditions can lead to extensive morbidity, mortality, and high health care costs. Currently, biopsy is the standard method for renal fibrosis staging. This method of diagnosis is painful, invasive, limited by sampling bias, exhibits inter- and intraobserver variability, requires prolonged hospitalization, poses risk of complications and significant bleeding, and could even lead to death. MRE based methods can potentially be useful to noninvasively detect, stage, and monitor renal fibrosis, reducing the need for renal biopsy. In this chapter, we describe experimental procedure and step by step instructions to run MRE along with some illustrative applications. We also includes sections on how to perform data quality check and analysis methods.

This publication is based upon work from the COST Action PARENCHIMA, a community-driven network funded by the European Cooperation in Science and Technology (COST) program of the European Union, which aims to improve the reproducibility and standardization of renal MRI biomarkers.
\end{abstract}

Key words Magnetic resonance elastography (MRE), Stiffness, Kidney, Preclinical imaging, MRI, Fibrosis, Chronic kidney disease (CKD)

\section{Introduction}

\subsection{Magnetic Resonance Elastography}

\begin{abstract}
Magnetic resonance elastography (MRE) based imaging techniques are now being routinely used for noninvasive assessment of tissue mechanical properties. In MRI, elastography encompasses imaging techniques that noninvasively estimate tissue elasticity and related mechanical properties through the application of external forces [1]. MRE induces harmonic vibrations of acoustic-range frequencies in the tissue of interest and images the propagation of these vibrations in the tissues to calculate quantitative values of mechanical parameters. The desired output is to measure the level of stiffness. The elastogram differentiate if the tissue is hard or soft can give diagnostic information about the presence of the disease. In
\end{abstract}


liver, tissue stiffness has been shown to be elevated with advanced fibrosis and to correlate with the stage of fibrosis [2-4]. Renal fibrosis is a pathological process common to all chronic kidney diseases (CKD) and thereby represents an excellent treatment target. A large number of molecular pathways involved in renal fibrosis have been identified in preclinical studies, some of them being similar among different organs and some with available drugs in various phases of clinical testing [5]. CKD may lead to end-stage renal failure, with extensive morbidity, mortality, and increasing health costs. Primary and secondary prevention requires a better knowledge of mechanisms underlying renal scarring, the development of specific therapies to slow down the progression of the disease and the development of noninvasive diagnostic tools to characterize the process.

Among imaging methods, MRE, that has been successfully demonstrated in the liver, is an attractive alternative for staging of renal fibrosis. Application of MRE to kidney has been shown to be possible and initial results are encouraging. MRE methods are advantageous as compared to ultrasound based elastography methods because the kidneys are usually located deeply in the body and therefore there is no direct access to apply an external compression easily. An increase in the extracellular matrix synthesis, with excessive fibrillary collagens, characterizes the development of chronic lesions in the glomerular, interstitial, and vascular compartments, leading progressively to end-stage renal failure [6]. Mechanisms participating in these processes are increasingly identified and various therapeutic interventions have been shown to prevent or to favor regression of fibrosis in several experimental models. Therefore, development of new noninvasive MRE based methods for identification and quantification of fibrosis is worthwhile.

\subsection{Modulus of Elasticity}

The shear modulus $(\mu)$ and Young's modulus $(E)$ are two parameters often used for describing tissue mechanical properties and are used as contrast parameters in elasticity imaging. Poisson's ratio $(\nu)$, another widely used parameter, is the ratio of lateral strain to longitudinal strain. Among these parameters, only two are independent. The shear modulus is known to be related to Young's modulus and one can be estimated from the other by knowing the Poisson's ratio. In general, soft tissues can be assumed to be incompressible and hence the Poisson's ratio can be assumed to be 0.5 . The relationship between shear modulus and Young's modulus can be written as $E=3 \mu$, which means that the calculation of Young's modulus or shear modulus provides the same information. MRE is a dynamic elasticity imaging technique capable of calculating quantitative values of shear modulus of tissues and is clinically approved to be used for the assessment of liver fibrosis. MRE uses low-frequency $(40-80 \mathrm{~Hz})$ sound waves to induce shear waves in the liver, visualizes the shear waves by tracking tissue displacement 
using a modified phase-contrast sequence, and measures the speed of the propagating wave with specialized software called an inversion algorithm [7]. In the commercial version of MRE supplied by Resoundant, the mechanical waves are generated by a subwoofer ("active driver") outside the scan room and are transmitted to a plastic disk ("passive driver") via a plastic tube passing through a wave guide [8]. For preclinical imaging, the same subwoofer active driver may be used or alternatively a functional generator may be used for generating a low frequency sinusoidal mechanical motion (typical range: 20-400 Hz). When the shear stresses (amplitude of microns) are applied to the top surface of a material with certain frequency, the induced waves are propagating into the tissue and every point in the tissue reciprocates in this micromotion. During clinical MRE image acquisition, the passive driver is secured by an elastic band over the abdominal organ of interest $[8,9]$. In general, most patients can feel the vibrations generated by the disk but do not find them uncomfortable. For clinical image postprocessing, identical MRE hardware and inversion algorithms are commercially available on scanners manufactured by major $\mathrm{MR}$ vendors $[10,11]$. Several studies have shown that MRE is a robust, reliable, repeatable, and reproducible technique for detection and staging of liver fibrosis [10]. The accuracy of MRE ranges from 0.85 to 0.99 for differentiating different stages of liver fibrosis [2]. The performance of MRE for differentiating mild fibrosis (stage I) from normal liver or inflammation is lower and the performance is highest for diagnosis of cirrhosis (stage 4) [4, 10]. For preclinical MRE, an actuator, replicating the use of passive driver may be used to couple to the tissue of interest to generate propagating shear waves within a soft tissue material [12]. The electrical signal for the drivers is created by a signal generator synchronized with the MRI pulse sequence triggered and is amplified by an audio amplifier before being fed into the mechanical driver. After the mechanical excitation, a phase-contrast based pulse sequence is used to encode the shear wave motion into the MR phase signal by using a series of magnetic field gradients called motion-encoding gradients (MEG). The MEG, synchronized with the mechanical excitations, is applied after RF excitation [13]. Postprocessing may be done on the scanner or images may be taken offline for analysis. Details of data processing and image reconstruction are covered in the following sections.

1.3 Need for Preclinical MRE
Renal fibrosis causes a change in the structure of the kidney wherein there is an excess accumulation of interstitial extracellular matrix and reduction in the number of tubules. Increasing evidence has demonstrated that, unlike cirrhosis, the early stages of fibrosis are treatable and reversible if appropriate antifibrotic treatment is given $[5,14]$. As antifibrotic therapies evolve, a reliable, noninvasive assessment of fibrosis is needed to manage patients with chronic 


\subsection{Quest for Renal MRE}

disease. Being able to noninvasively monitor the progression of fibrosis would help in understanding the natural history in patients with chronic disease, determining which patients require antiviral therapy, predicting the approximate time to the development of high-grade fibrosis, and discovering new directions of scientific inquiry. Therefore, there are ongoing investigations using preclinical models with a reliable, noninvasive method to assess organ inflammation and fibrosis, not only to detect and stage the disease itself but also to monitor treatment efficacy and optimize dosing. The feasibility of MRE has been demonstrated on a mouse model of autosomal recessive polycystic kidney disease (ARPKD) which is an inherited disorder of the kidneys and liver caused by mutations in the PKHDl gene and an important cause of congenital hepatic fibrosis in humans [3].

Many different mechanisms induce glomerular injury, including glomerulonephritis, hypertensive nephrosclerosis and diabetic nephropathy. However, once renal damage reaches a certain threshold, progression of renal disease is consistent, and largely independent of the initial insult. This common pathway to end-stage renal failure is mainly due to tubulointerstitial damage characterized by tubular atrophy, loss of peritubular capillaries and interstitial fibrosis. Mechanisms leading to kidney failure via tubulointerstitial damage and development of fibrosis are mostly massive proteinuria and chronic hypoxia. Fibrosis further impairs oxygen diffusion and supply to tubular cells, rendering a vicious cycle. Thus, in the assessment of chronic renal failure, fibrosis is a major histological feature and may be an important surrogate endpoint for prognosis and monitoring of treatment response. Besides, some investigations suggest that fibrosis might be reversible, when the cause is treated, emphasizing the need for an early detection and quantification of this fibrosis [14-19]. Currently, the extent of interstitial fibrosis is evaluated by renal biopsy which has several drawbacks: it is a semiquantitative approach with wide interobserver variability, and it is prone to sampling errors and associated with significant morbidity. Thus a noninvasive, truly quantitative method of interstitial fibrosis monitoring would be desirable. Diffusion-weighted and blood oxygenation level dependent (BOLD) MRI findings have been shown to be correlated to renal function, but no data have been published as to their correlation with the degree of fibrosis. Blood or urinary markers of fibrosis are also currently evaluated but none is used in clinical practice yet. MRE based methods can estimate tissue stiffness (and thus, the degree of fibrosis) by measuring the velocity of shear waves traveling through the organ of interest. Clinically, they have been mostly used in liver. The ultrasound-based transient elastography (Fibroscan ${ }^{\mathrm{TM}}$ device) is able to discriminate the different stages of liver fibrosis with a quadratic trend of the curve plotting histologic scores versus 
elasticity measurements. Among patients with cirrhosis, stiffness thresholds predicting the onset of specific complications (ascites, esophageal bleeding, hepatocarcinoma, etc.) have been identified. However, this technique is limited by its ID nature that does not allow the exploration of the entire liver. MRE has been successfully used to assess liver fibrosis. The shear stiffness of normal liver was found to be approximately $2.2 \mathrm{kPa}$ by independent groups, using vibrating frequencies of $60 \mathrm{~Hz}$ [4]. As with ultrasound-based transient elastography, MRE can discriminate the different stages of liver fibrosis, with the same quadratic trend of the fibrosis/elasticity curve [2]. MRE has the advantage of being intrinsically a 2D technique and can be associated with conventional liver imaging at the same time. MRE has also been used for applications in the abdomen such as characterizing focal liver lesions, and in organs such as pancreas, breast, muscle, prostate, and kidney [9].

Currently, the most definitive means of assessing renal fibrosis is a kidney biopsy. The invasiveness of a biopsy coupled with its small sampling size have limited its utility in quantifying and monitoring changes in renal fibrosis, especially because fibrosis can be heterogeneously distributed. Accordingly, a noninvasive modality that assesses fibrosis on a kidney-wide scale would be of clinical utility. By replacing soft healthy tissue with stiff extracellular matrix, fibrosis stiffens organs, a phenomenon that has led multiple groups to explore whether tissue stiffness could be used as a surrogate measure for fibrosis. MRE based technique that measures tissue stiffness, would be a novel application for assessment of renal fibrosis. During MRE, the organ of interest is gently vibrated by applying acoustic waves through a pad placed on the overlying skin. The resulting microscopic vibrational waves passing through the organ generate shear waves that can be imaged with motion-synchronized MRI. The velocity of wave propagation is dependent on organ stiffness, with stiffer fibrotic tissue leading to more rapidly moving waves with longer wavelength. Capturing shear waves at multiple time points enables the reconstruction of a quantitative stiffness map. MRE is already a well-established, gold standard technique for liver fibrosis imaging, having been shown to accurately reflect biopsy-derived liver fibrosis measurements. In contrast, MRE for nonhepatic applications is still early in its development, and its technique has not yet been standardized. Small pilot studies of MRE have been performed in native kidneys and kidney allografts, attempting to correlate kidney stiffness with either fibrosis burden or kidney function. Although MRE in porcine kidneys showed a correlation between stiffness and fibrosis in the medulla, the results of small pilot human studies thus far have been conflicting, and no studies as yet have assessed whether stiffness measured by MRE predicts progression of kidney dysfunction. In this chapter, we provide recommended MRE protocols for applications in the abdomen with a focus on the kidney, and a few illustrative applications of MRE in preclinical imaging. 
This introduction chapter is complemented by a separate chapter describing the concepts of MRE in the abdomen, which is part of this book.

This chapter is part of the book Pohlmann A, Niendorf T (eds) (2020) Preclinical MRI of the Kidney-Methods and Protocols. Springer, New York.

\section{Materials}

\subsection{Animals}

\subsection{Lab Equipment}

MRE is an MR-based elastography technique that uses mechanical shear waves to evaluate viscoelastic properties of tissues. Mechanical shear waves produced by an external driver are propagated into the organ of interest using a passive abdominal driver placed over the organ and in contact with the abdominal wall. Tissue displacements in the range of microns produced by propagating shear waves because phase shifts that can be detected with motion encoding gradients in the MRE sequence. The frequency of shear wave applied ranges from 40 to $400 \mathrm{~Hz}$. In liver, the frequency of shear waves used for clinical liver MRE is $60 \mathrm{~Hz}$. A lower frequency is used for organs that are situated deep in the body and a higher frequency may be used for regions of interest that may be closer to the body surface. A lower frequency wave has deeper penetration and spatial lower resolution. A higher frequency wave has higher attenuation and higher spatial frequency. Once decided on the resolution and frequency, these parameters must stay consistent for the duration of the study and must not be changed. Stiffness values measured with one particular frequency are not convertible with those measured with a different frequency. An inversion algorithm automatically produces stiffness maps using the phase shift information. Shear stiffness values can be obtained by drawing regions of interest over the tissue of interest in the stiffness maps and expressed in kiloPascals $(\mathrm{kPa})$.

The experimental protocols in this section are tailored for rats (Wistar, Sprague-Dawley or Lewis) with a body mass of 250-350 g. Advice for adaptation to other smaller animals (e.g., mice) or larger animals (e.g., pigs) are given in the sections where necessary.

1. Anesthesia: For nonrecovery experiments urethane solution (Sigma-Aldrich, Steinheim, Germany; 20\% in distilled water) can provide anesthesia for several hours with comparatively few side effects on renal physiology, which is an important issue. For an in-depth description and discussion of anesthesia please refer to the chapter by Kaucsar $\mathrm{T}$ et al. "Preparation and Monitoring of Small Animals in Renal MRI."

2. Gases: $\mathrm{O}_{2}, \mathrm{~N}_{2}$, and compressed air, as well as a gas-mixing system (FMI Föhr Medical Instruments $\mathrm{GmbH}$, SeeheimOber Beerbach, Germany) to achieve required changes in the 
oxygen fraction of inspired gas mixture $\left(\mathrm{FiO}_{2}\right)$. Besides air, the following gas mixtures are required during the experiment: $10 \% \mathrm{O}_{2}-90 \% \mathrm{~N}_{2}$ for hypoxia and $100 \% \mathrm{O}_{2}$ for hyperoxia.

3. Device for $\mathrm{FiO}_{2}$ monitoring in gas mixtures: for example Capnomac AGM-103 (Datex GE, Chalfont St Gils, UK).

\subsection{MRI Hardware}

2.3.1 Generating Propagating Shear Waves in the Abdomen: Hardware
The general hardware requirements for renal $1 \mathrm{H}$ MRI on mice and rats are described in the chapter by Ramos Delgado P et al. "Hardware Considerations for Preclinical Magnetic Resonance of the Kidney." The technique described in this chapter is tailored for a standard 1.5 T or a 3.0 T MR scanner using a head coil or small flex coil (for signal reception) or knee RF coil (transmit-receive) is given where necessary but advice for adaptation to other field strengths and systems (e.g., 9.4 T MR system Biospec 94/20, Bruker Biospin, Ettlingen, Germany). No special or additional hardware is required, except for the following:

1. A physiological monitoring system that can track the respiration, and which is connected to the MR system such that it can be used to trigger the image acquisition.

2. MRE hardware to generate shear waves in the abdomen, details explained in the following section. The experimental protocols are tailored for using a FDA approved and commercially available subwoofer-based system for generating shear waves (Resoundant, MN, USA). Advice for options on using other in-house custom-made MR conditional hardware is included where necessary.

MRE has received considerable attention due to the flexibility of $\mathrm{MR}$ in acquiring arbitrary 2D images and 3D volumes and its capability to measure motion in all three directions. Though the application of excitation to the tissue of interest could be either internal or external, and either static or dynamic, MRE typically uses dynamic vibrations of a single frequency (within the audio frequency range) induced by external drivers. The electrical signal for these drivers is created signal generator synchronized with MRI pulse sequence triggered and is amplified by an audio amplifier before being fed into the mechanical driver. The mechanical vibrations applied to tissue fall into three categories: transient, quasistatic, or harmonic. It is now well known that the MRE-measured shear modulus of soft tissue is dependent on the frequency of applied mechanical waves. That is why the term "shear stiffness" is often used to describe the shear modulus at a specific frequency. However, it should be fully understood that the formula that calculates the shear modulus from the measured velocity of the waves $\left(\mu=\rho c^{2}\right.$, where $\mu=$ shear modulus, $\rho=$ density of tissue, and $c=$ wave speed) is valid only in purely elastic tissues. MRE 

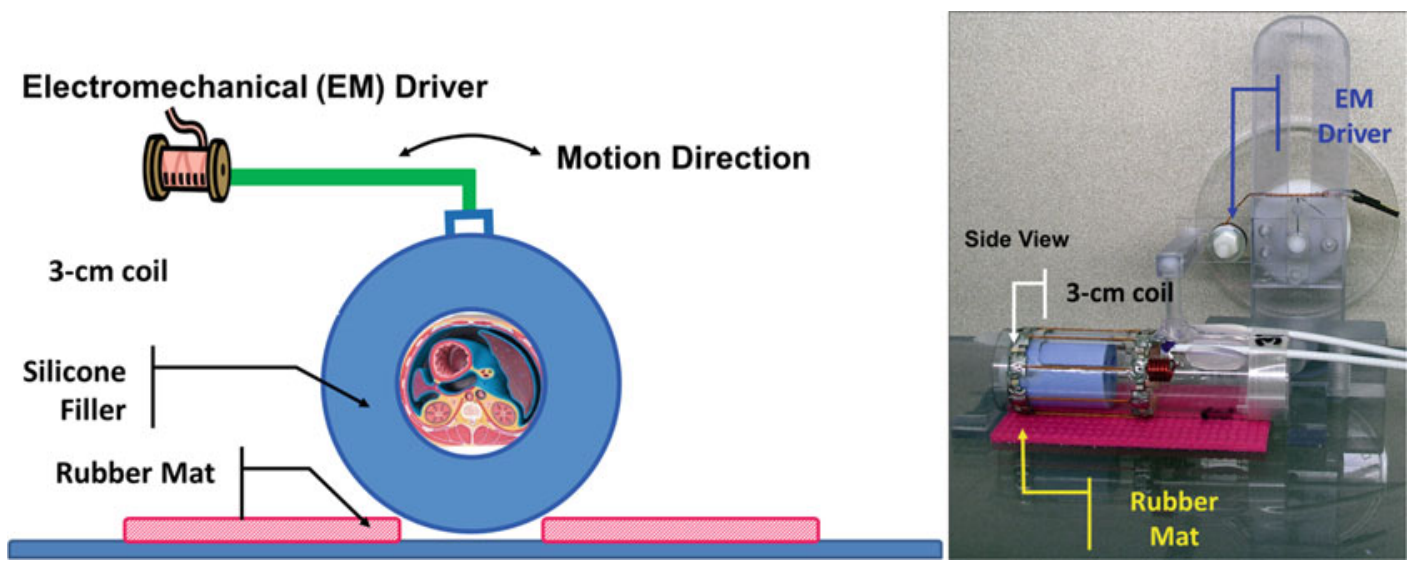

Fig. 1 For smaller animals, an electromechanical driver setup may be used for shear wave generation. Rotational vibrations generated by the electromechanical driver are transmitted into the entire body of the animal. The transmitted shear wave frequency range can be set between 50 and $400 \mathrm{~Hz}$

typically uses harmonic vibrations generated by an external driver device to perturb the tissue. In harmonic excitation, a low-frequency acoustic wave $(20-400 \mathrm{~Hz})$ is transmitted within the tissue using a sinusoidal mechanical source. When performing MR elastography, the goal is to acquire tissue stiffness maps for each imaging slice, with a large area of the tissue of interest not covered by the $95 \%$ confidence map so that a large portion of the tissue can be measured. The confidence maps are calculated during the stiffness inversion, as the fit of a smooth polynomial to the phase images. In a typical MR elastography configuration, to create a sinusoidal mechanical motion, an active pneumatic wave driver, which is a subwoofer, is located outside the MR scanner room and is connected, by way of a flexible plastic (polyvinyl chloride) tube, to a passive driver that is fastened onto the abdominal wall or the organ or interest. For the most part, three kinds of active driver mechanical driving mechanisms have been used in the literature. They are electromechanical drivers, piezoelectric drivers, and acoustic speaker-based driver systems [20-22]. Electromechanical drivers were one of the initially developed driver systems (Fig. 1). They use the Lorentz force between the induced magnetic moment created by voice coils, and the static magnetic field of the main MRI magnet to create the motion [20]. Piezoelectric and needle-based drivers have commonly been used in preclinical or MRE of tissues $[12,21]$. This is primarily because of the advantages of the ease of positioning of the driver in comparison to the electromechanical drivers and the avoidance of an additional magnetic field, leading to a reduction of artifacts [22]. Also, these drivers can create more reliable waveforms with less ringing effects, which becomes especially useful in situations where a single transient wave needs to be induced in the tissue or sample of interest. Acoustic speaker-based 
driver systems are commercially available and are commonly used for clinical MRE scans. The vibrations in the acoustic systems are again produced by the Lorentz force, but the static magnetic field is from a devoted permanent magnet present in an acoustic speaker. These speakers, with their own permanent magnets, that are much weaker than the static field $B_{0}$ of the MR system, are designed such that they are placed away from the main magnet. Thus, this system necessitates an additional component to couple the vibrations produced by the speakers to the tissue of interest. The active driver is coupled to a passive driver that is positioned on the abdominal organ of interest. The passive driver transfers a continuous acoustic vibration that is transmitted through the entire abdomen, at a fixed low frequency, which is typically $60 \mathrm{~Hz}$ for the liver. Choice of frequency is a balance between wave penetration and desired spatial resolution. A higher frequency improves spatial resolution while a low frequency improves wave penetration. The advantage of this system is that the passive drivers can be developed according to the application in different organs and can thus be used to effectively propagate shear waves within the desired organ of interest with comfort to the patient. For preclinical images in small animals such as mice or rats, a frequency of up to $400 \mathrm{~Hz}$ may be used to get the desired spatial resolution. A modified phase-contrast based pulse sequence with motion encoding gradient pairs synchronized to oscillate at the same frequency of mechanical waves created by the passive driver are used to encode the motion of the tissues in the phase of an MR image. This sequence is then used to image the micron-level cyclic displacements caused by the propagating shear waves to create a magnitude image, which provides anatomic information, and a phase image, which provides wave motion information. The phase or "wave" images, containing wave-propagation information, are then inverted to calculate quantitative stiffness maps (elastograms).

2.3.2 Pulse Sequence for MRE Motion Encoding: Software
The two main pulse sequence groups in MRI are spin echo (SE) and gradient recalled echo (GRE). In the early 1980s, the SE sequence was the main magnetic resonance sequence in clinical imaging [23]. Later, the GRE sequence proposed by Mansfield and Maudsley became an essential technique and a good alternative to SE sequences [24]. The two types of acquisition sequences currently in use to obtain abdomen organ stiffness values are the GRE based and the SE based with echo-planar readout; both of which have been shown to have excellent correlation on both 1.5 and $3.0 \mathrm{~T}$ MRI scanners [13, 25, 26]. The use of GRE-based sequence has demonstrated to correlate with histological grading of liver fibrosis in previous studies and a recent meta-analysis [10, 13]. However, the inherent limitations of GRE-based acquisition on field strengths of $3.0 \mathrm{~T}$ or higher in addition to enhanced sensitivity to 
susceptibility, is the signal drop off due to longer echo time (TE) and relatively shorter $\mathrm{T} 2$ relaxation time of the liver. GRE-based MRE also has relatively lower accuracy in obese patients due to the increased distance from the driver to the liver. Thicker layers of fat can result in signal loss and produce limitations for encoding shear waves in the deeper areas of the liver, hence reducing the resulting stiffness measurable area [25]. Since chronic liver disease is quite frequently associated with obesity, radiologists should be aware of the limitations of GRE-based acquisition in such cases. GRE-based MRE acquisition also require relatively longer periods of acquisition and have higher susceptibility to breathing motion. Clinically, the current GRE-based protocol, for MRE image acquisition requires a breath-hold of approximately $20 \mathrm{~s}$ for a single slice. Echo-planar imaging (EPI), on the other hand, is a fast magnetic resonance imaging technique that obtains all spatialencoding information in a single radiofrequency (RF) pulse, allowing for faster acquisition times with less motion artifacts. SE-EPI MRE has several advantages over the GRE MRE sequence: (1) It allows for acquisition of multiple slices in a single breath-hold covering entire kidney as compared to GRE MRE, which allowed for acquisition of only a single slice per breath-hold; (2) Entire 3D acquisition with three motion encoding directions can be performed in three breath-holds, which enables completion of the study in patients who cannot perform more than three breathholds and cannot lay flat for longer period of time in the scanner; (3) SE-EPI MRE can avoid problems such as data misregistration caused due to multiple breath-holds when acquiring multiple slices compared to GRE MRE. SE-EPI-based images are also advantageous because it enables measuring larger areas of the organ due to higher waves encoded per relaxation time (TR). Despite these advantages, use of SE-EPI MRE sequence has a few limitations such as it is prone to the distortion artifacts arising from the eddy currents caused by gradients switching on and off rapidly. These distortions may cause increase in the apparent wavelength of the waves propagating through the kidneys thereby causing an increase in the stiffness estimations. Care must be taken to correct for the distortions when observed. Additionally, SE-EPI MRE is also very sensitive to chemical shift and ghosting artifacts. Appropriate steps need to be taken during acquisition by application of spatial spectral frequency pulses and phase correction to correct for these artifacts, respectively for accurate estimate of MRE-derived stiffness values.

The tissue response to the applied harmonic excitation is represented in terms of waves propagating within the tissue. After the mechanical excitation, the phase-contrast based pulse sequence is used to encode the shear wave motion into the MR phase signal by using a series of magnetic field gradients called MEG. The MEG is typically applied after RF excitation pulses and before signal 


\subsection{Image Postprocessing: Generating Elastograms for Stiffness Estimation}

\subsection{Periodic Quality Assurance (QA)}

acquisition. From the acquired wave images, inverse reconstruction algorithms are applied to convert the displacement data to mechanical properties [27]. The mechanical quantity that MRE characterizes typically is shear modulus $(\mu)$, which describes the proportionality relationship between the shear stress and strain. In MRE excitation is primarily shear and the displacements in MRE are very small ( microns). Therefore, it is a good approximation to model tissue as a linear, viscoelastic solid. It can be assumed that there is no volume change as layers of material move in shear, perpendicular to the direction of the wave propagation. The shear stiffness, $\mu$, can be calculated if the wavelength, $\lambda$, is measured, as the frequency is known and the density of most soft tissues is close to the density of water $\left(1000 \mathrm{~kg} / \mathrm{m}^{3}\right)$. Assuming an isotropic material, this reduces to two independent quantities, the Lame constants $\lambda$ and $\mu$ related to longitudinal and shear deformation respectively. If local homogeneity is assumed, $\lambda$ and $\mu$ become single unknowns, and the equation for harmonic motion becomes an algebraic matrix equation that can be solved locally: $\mu \nabla 2 u+(\lambda+\mu) \nabla(\nabla \cdot u)=-\rho \omega 2 u[28]$, with $\rho$ the density, $\omega$ the angular frequency of the mechanical oscillation, and $u$ the displacement vector. Approaches such as local frequency estimation (LFE), phase gradient (PG), and direct inversion (DI) have been used to estimate the localized wavelength $[28,29]$. The algorithms assume that the waves are propagating in a uniform, infinite, homogeneous medium. The LFE algorithm can estimate the spatial frequency of the propagating shear waves. LFE is a robust method for estimating isotropic tissue stiffness even in the presence of noise when compared to other methods, as it is performed in the k-space domain. However, it suffers from edge effects where the estimates values at the sharp boundaries can be inaccurate. Hence, if LFE is used, the ROIs on the elastograms must be drawn away from the edges. DI is another inversion algorithm where lower order polynomials are fit to the displacement field data to obtain its Laplacian; and solving the Helmholtz wave equation by plugging the Laplacian generates the stiffness map. However, DI is also sensitive to noise because of the Laplacian operator.

Recommended QA: Measurements of organ stiffness (magnitude of the complex shear modulus) obtained with MRE depend on the spatial fidelity of the acquired phase images. Therefore, the validity of the field of view and image linearity should be assessed and confirmed on an ongoing basis, as is already routine for all clinical scanners, using manufacturer-recommended procedures.

While other instrumental causes of drift in stiffness measurements have not been documented in the literature, technical failures such as faulty synchronization of the driver system or incorrect driver frequency settings can cause incorrect measurement. 
Performing and documenting a routine QA process also helps in preparation of local institutional research guidelines.

Optional QA: Correct user setup and proper functioning of the MRE system can be confirmed using a phantom with known stiffness properties. These usually consist of a uniform, tissuesimulating material with known stiffness and known stability over time and storage conditions. An MRE phantom can be used to confirm proper functioning of the MRE system after initial installation and as a periodic test of correct functioning. There is as yet no consensus on recommendations for the frequency of phantom testing. Optional QA testing with a phantom should employ a protocol recommended by the phantom manufacturer.

\section{Methods}

\subsection{Animal Setup on the Scanner}

1. As described in Subheading 2, in a typical preclinical MRE study, animals are anesthetized by intraperitoneal injections of ketamine hydrochloride $(50 \mathrm{mg} / \mathrm{kg}$ ) and xylazine hydrochloride $(5 \mathrm{mg} / \mathrm{kg})$.

2. After shaving and preparation of the abdomen, animals are placed and fixed on a plastic table in the supine position. It is important to position the animal as close to the isocenter as possible to avoid any $B_{0}$ or $B_{1}$ inhomogeneity artifacts.

3. Start the temperature monitoring system, apply some surgical lubricant to the temperature probe and place it in the rectum of the animal.

4. Attach the respiration sensor to the upper abdomen of the animal using adhesive tape. Start and setup the respiratory monitoring system. If necessary, adjust the position of the respiration sensor until the amplitude of the respiration trace is sufficiently large for the system to reliably detect the trigger points at the beginning of expiration.

5. A silver needle (disposable silver acupuncture needles, Asahi Medical Instrument Co., Kawaguchi, Saitama, Japan) can be used in lieu of a passive driver and gently inserted gently into the target tissue through the anterior body wall (Figs. 2 and 3). The other end of the needle is attached to an electromechanical driver that generated longitudinally orientated sinusoidal vibrations at low frequency.

1. MR image acquisitions may be performed with a standard 1.5 $\mathrm{T}$ or a $3.0 \mathrm{~T}$ whole body MRI scanner using a receiving surface coil.

2. Acquire a fast pilot scan to obtain images in the three orthogonal planes $x, y$, and $z$. 

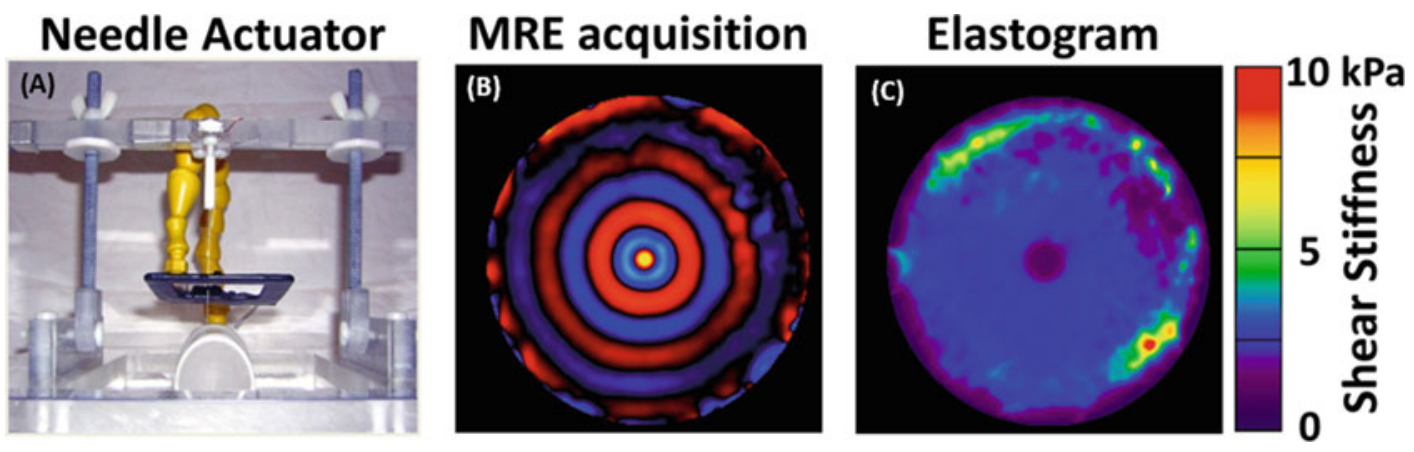

Fig. 2 (a) Drawing illustrates a typical experimental setup of a mouse or rat MR elastography configuration. A passive driver, a needle, is used to generate circular shear wave fields. A plastic connecting tube connects the passive driver to the active driver, which is located behind a wall outside the imaging room. This setup is relatively easy to apply for preclinical imaging. The active driver used may be a standard commercially available equipment. (b) Images acquired during a MRE phantom scan. Magnitude and phase images yield raw data: The magnitude images provide anatomic information, and the phase images provide wave motion information. After postprocessing, color wave images and (c) stiffness maps, also known as elastograms, are generated

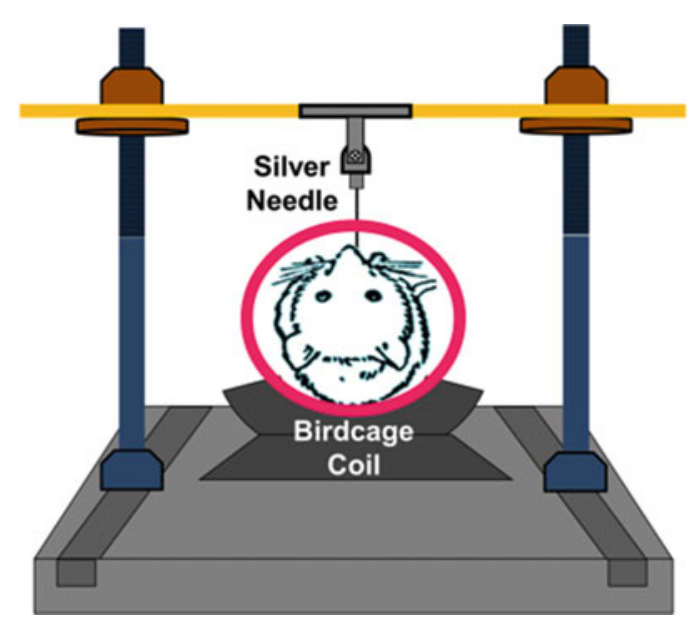

Fig. 3 A typical experimental setup for a mouse MRE study. A silver needle is used to generate circular shear wave field in the mouse liver in vivo

3. Acquire anatomical images in several oblique orientations to facilitate planning a coronal slice orientation with regards to the long axis of the kidney, as described in the chapter by Pohlmann A et al. "Essential Practical Steps for MRI of the Kidney in Experimental Research."

4. Perform localized shimming on the kidney as described in the chapter by Pohlmann A et al. "Essential Practical Steps for MRI of the Kidney in Experimental Research" (see Note 16). 
Table 1

Recommended 2D MRE protocol parameters

\begin{tabular}{lll}
\hline Parameter & 2D GRE MRE & 2D SE-EPI MRE \\
\hline TR $(\mathrm{ms})$ & 50 & 1000 \\
\hline TE $(\mathrm{ms})$ & 23.7 & 30 \\
\hline Matrix size & $128 \times 64$ interpolated to $128 \times 128$ & $100 \times 100$ (true) \\
\hline Voxel size $(\mathrm{mm})$ & $1.2 \times 1.2$ & $1.5 \times 1.5$ \\
\hline Slice thickness $(\mathrm{mm})$ & 6 & 6 \\
\hline Bandwidth $(\mathrm{Hz} / \mathrm{Px})$ & 399 & 2380 \\
\hline No. of averages & 1 & 1 \\
\hline No. of slices & 4 & 4 \\
\hline MEG frequency $(\mathrm{Hz})$ & 60 & 60 \\
\hline MEG direction & $Z$ axis $($ slice $)$ & $Z$ axis (slice) \\
\hline Echo spacing $(\mathrm{ms})$ & 24.7 & 0.5 \\
\hline EPI factor & NA & 100 \\
\hline Acceleration factor & 2 & 2 \\
\hline Scan time (min: s) & $1: 00$ & $0: 11$ \\
\hline
\end{tabular}

\subsection{MRE Imaging}

1. A gradient echo (GRE) MRE sequence may be used for wave image acquisition with the following parameters: matrix size of $256 \times 64, \mathrm{TR} / \mathrm{TE}$ of $33.3 \mathrm{~ms} / 17.8 \mathrm{~ms}$, flip angle of $30^{\circ}$, slice thickness of $5 \mathrm{~mm}$, field of view of $10 \mathrm{~cm} \times 10 \mathrm{~cm}$, one pair of $1.76 \mathrm{G} / \mathrm{cm}$ trapezoidal motion-encoding gradients (MEG) at $120 \mathrm{~Hz}(8.33 \mathrm{~ms}$ duration). The repetition time is typically set as an integer multiple of the mechanical wave period to allow for continuous shear wave motion during the scan via the customary synchronized trigger pulses. Details of protocol parameters for relevant acquisition sequence choices are provided in Tables 1, 2 and 3.

2. It should be noted that the trigger pulses are moved during the scan to obtain images at four different phase offsets between the motion and the MEG, equally spaced over one cycle of the motion.

3. Perpendicular to the driving needle, several sequential imaging planes containing tissue of interest should be chosen for MRE wave data collection. The number of slices to be acquired depends on the size of the organ of interest, but is typically $2-4$.

4. The MEG should be set to sensitize the shear motion along the through-plane direction (i.e., the anterior-posterior or sliceselect direction for these coronal images). Typically, respiratory 
Table 2

Recommended imaging protocol for propagating shear waves in the abdomen (small animal 3D MRE protocol parameters)

\begin{tabular}{|c|c|}
\hline Parameter & 3D MRE \\
\hline Pulse sequence type & 2D SE-EPI \\
\hline Matrix & $128 \times 128$ \\
\hline No. of averages & $1-16$ \\
\hline No. of EPI shots & 1 \\
\hline $\mathrm{TR}(\mathrm{ms})$ & 1000 \\
\hline $\mathrm{TE}(\mathrm{ms})$ & Minimum \\
\hline No. of slices & $12-20$ \\
\hline Slice thickness (mm) & 1 \\
\hline Gap (mm) & 0, Interleave \\
\hline No. of phases & $3-4$ \\
\hline MEG frequency $(\mathrm{Hz})$ & $200-400$ \\
\hline MEG direction & $X, \Upsilon$, and $Z$ axes \\
\hline MEG Power per axis & $25 \mathrm{G} / \mathrm{cm}$ \\
\hline Field of view $(\mathrm{cm})$ & $3-4$ \\
\hline \multicolumn{2}{|c|}{$\begin{array}{l}\text { If available on the scanner, it is strongly recommended to use } 3 \mathrm{D} \text { MRE protoco } \\
\text { parameters } \\
G R E \text { gradient recalled echo, } S E \text { - } E P I \text { spin-echo echo-planar imaging, } 2 D \text { two dimen- } \\
\text { sional, } M R E \text { magnetic resonance elastography, } M E G \text { motion encoding gradients, } m \text { se } \\
\text { milliseconds, } m m \text { millimeters, } H z \text { hertz, } m T / m \text { milliTesla/meter, } P x \text { pixel, min minutes } \\
s \text { seconds, } N A \text { not applicable }\end{array}$} \\
\hline
\end{tabular}

or cardiac gating is not needed for lower abdomen organs such as liver and kidneys but this may be added if significant motion is observed. With a fixed needle inserted into the liver tissue, anaesthetized animals only performed shallow and fast upperchest breathing instead of deep and slow diaphragm breathing. Therefore, the tissue do not move much during respiration. In addition, the phase-encoding direction is chosen to be rightleft (frequency-encoding direction superior-inferior), which prevents possible motion artifacts from the chest wall and the heart from corrupting the tissue signal. In preclinical setting, motion artifacts can also be minimized by using multiple averages. As a reference for mice MRE imaging, preclinical MRE studies on ten ARPKD mice, with ages ranging from 3 to 12 months were used in the study by Yin et al. [3].

An accurate and reproducible stiffness measurement of the kidney, given its small size, and deeply seated location, requires 
Table 3

Recommended 3D MRE imaging protocol parameters for clinical renal imaging

\begin{tabular}{|ll}
\hline Parameter & 3D MRE \\
\hline Pulse sequence type & 2 D SE-EPI \\
\hline Matrix & $96 \times 96$ \\
\hline No. of averages & 1 \\
\hline No. of EPI shots & 1 \\
\hline TR $(\mathrm{ms})$ & 2400 \\
\hline TE $(\mathrm{ms})$ & Minimum \\
\hline No. of slices & 42 \\
\hline Slice thickness $(\mathrm{mm})$ & 3.6 \\
\hline Gap (mm) & 0, Interleave \\
\hline No. of phases & 3 \\
\hline MEG frequency $(\mathrm{Hz})$ & 120 \\
\hline Wave frequency $(\mathrm{Hz})$ & $60 / 90$ \\
\hline MEG direction & $X, \Upsilon$, and $Z$ axes \\
\hline No. of breath-holds & 6 \\
\hline Acceleration factor & 3 \\
\hline Driver amplitude range & $30-50 \%$ \\
\hline
\end{tabular}

3D analysis of wave field data. For this reason, if available on the scanner, 3D MRE is recommended to be utilized for renal imaging. In $3 \mathrm{D}$ MRE, the propagating shear waves are imaged with a $2 \mathrm{D}$ multislice spin-echo echo-planar imaging (SE-EPI) pulse sequence modified to include the motion encoding gradients (MEG) in the $X, \Upsilon$, and $Z$ directions. MEGs can be applied in one or more directions to encode the motion in those specific directions. This is different from the 2D MRE typically utilized for commercial MRE imaging in which motion is encoded only in a single direction.

A typical small animal preclinical 3D MRE protocol is shown in Table 2.

Figures 4, 5, and 6 show cross sectional spin echo anatomical images of the animal abdomen, more specifically of the mouse liver region and its respective wave displacement images along the $x, y$, and $z$ directions and the absolute values of the complex shear modulus.

In 3D MRE, the propagating shear waves are imaged with a $2 \mathrm{D}$ multislice spin-echo echo-planar imaging (SE-EPI) pulse sequence 


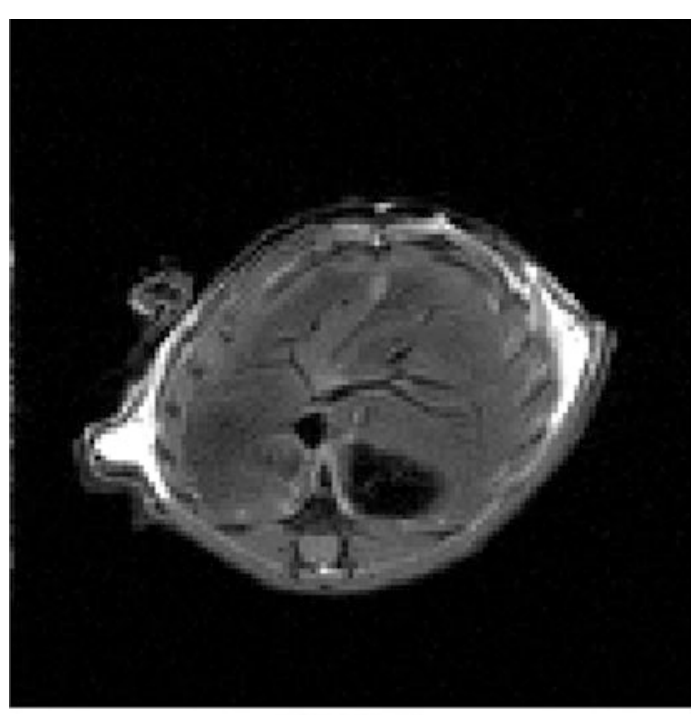

CONTROL

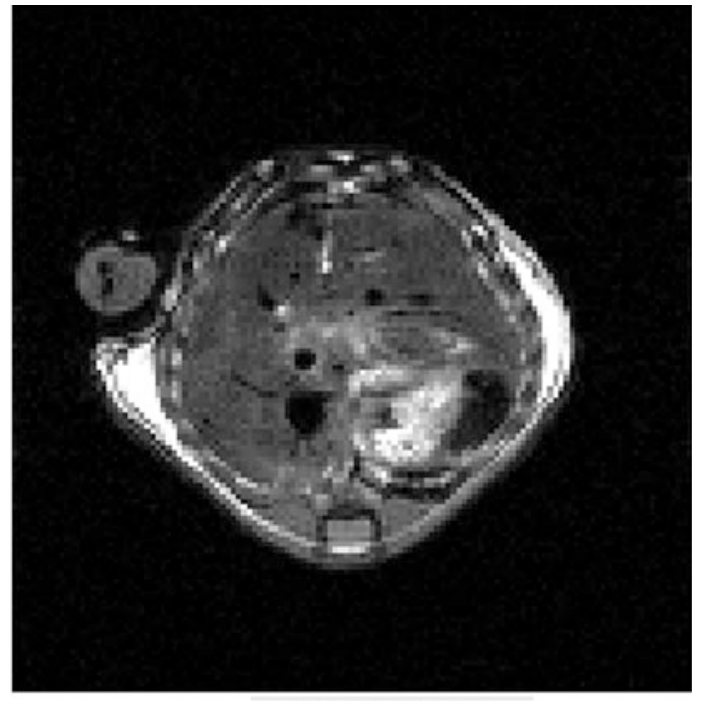

FIBROSIS

Fig. 4 Cross-sectional spin-echo anatomical images of the mouse abdomen, more specifically of the liver region (Image courtesy of Dr. S. Majumdar and Dr. D. Klatt from the Motion-encoding MRI Lab at the University of Illinois at Chicago, Chicago, Illinois, USA)

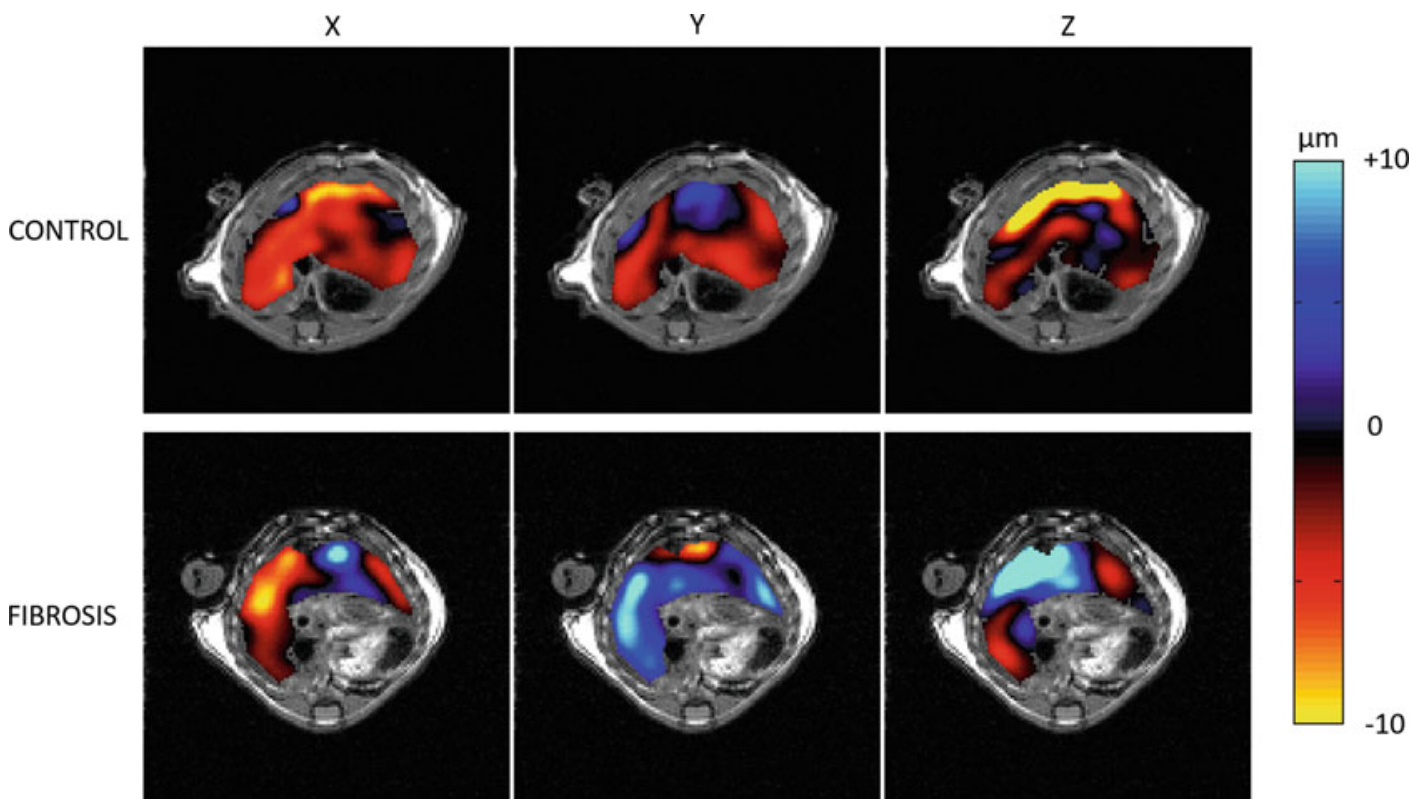

Fig. 5 Wave-displacement images along the $x, y$, and $z$ directions (Image courtesy of Dr. S. Majumdar and Dr. D. Klatt from the Motion-encoding MRI Lab at the University of Illinois at Chicago, Chicago, Illinois, USA)

modified to include the motion encoding gradients (MEG) in the $X, \Upsilon$, and $Z$ directions. Since kidney is a viscoelastic material, the stiffness varies with excitation frequency (i.e., stiffness increases with an increase in frequency). Once decided, it is important to keep the MEG frequency consistent for the duration of the study. 


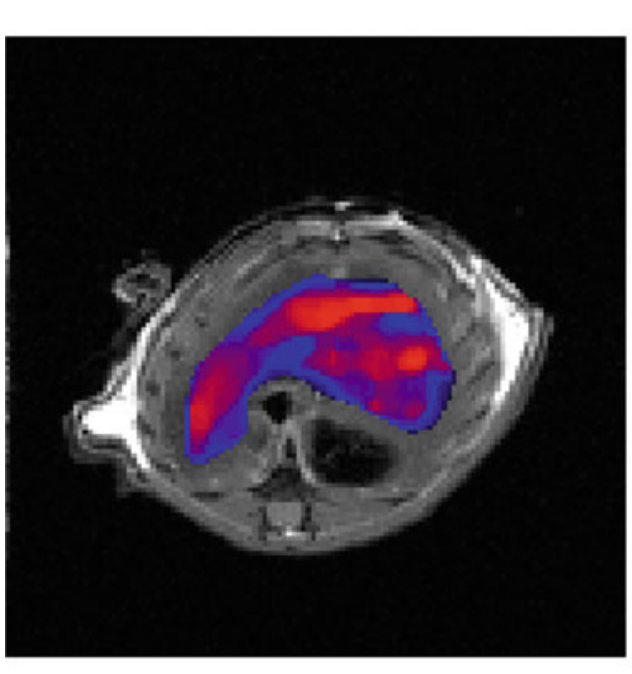

CONTROL

$3.55 \mathrm{kPa}$
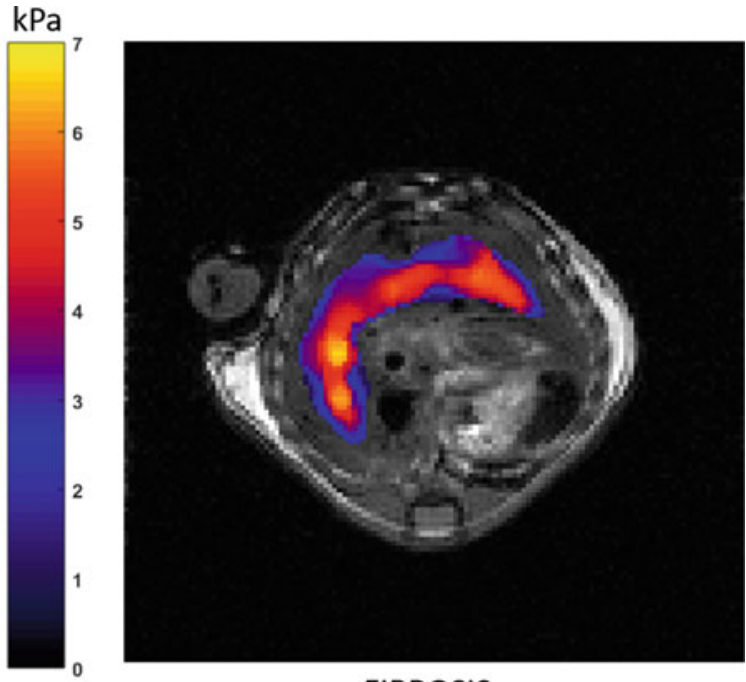

FIBROSIS

$4.19 \mathrm{kPa}$

Fig. $6\left|G^{\star}\right|$ or the absolute value of the complex shear modulus (Image courtesy of Dr. S. Majumdar and Dr. D. Klatt from the Motion-encoding MRI Lab at the University of Illinois at Chicago, Chicago, Illinois, USA)

\subsection{Image Review to Ensure Technical Success}

At a frequency of $60 \mathrm{~Hz}$, the average kidney stiffness range of $3.5-5 \mathrm{kPa}$ has been reported on normals in the literature by multiple groups $[30,31]$. At a frequency of $90 \mathrm{~Hz}$, a higher kidney stiffness range of 5-7.5 kPa was measured.

The raw magnitude and phase images obtained from the MRE acquisition should be reviewed on the scanner console at the time of the exam. The magnitude images should show signal loss in the subcutaneous fat just below the passive driver placement, confirming that mechanical waves are being applied. The signal loss is due to the phase images (also known as wave images) should demonstrate shear waves in the specific abdominal organ of interest. In the color phase images, red to blue region is a wavelength and this wavelength becomes longer in the presence of a stiff region for a given excitation frequency. Then these wave images are converted into spatial stiffness maps using an inversion algorithm known as elastograms. If no waves are seen in the tissue of interest, then the driver system should be checked. A phantom scan of known stiffness may be performed if needed to validate driver system. Shear wave images represent an instantaneous snapshot of the mechanical waves propagating through the tissue. After the magnitude and phase images are created, an inversion algorithm is applied to processes these raw data images to create several additional images and maps. 

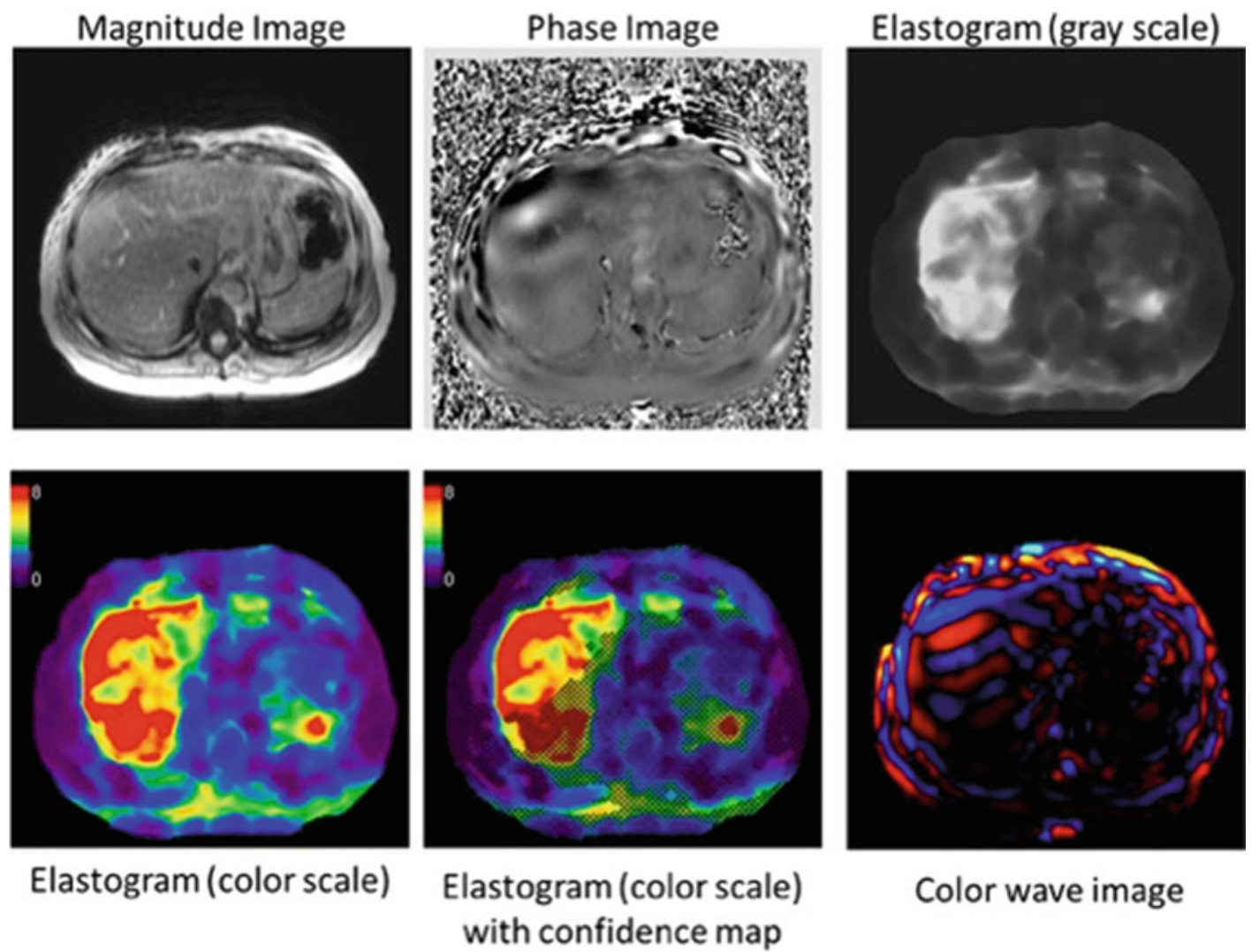

\section{Color wave image}

Fig. 7 Representation of typical postprocessed images generated in a clinical liver MRE study. Additional postprocessed images may be generated offline depending on the software version of the pulse sequence installed on the scanner

\subsection{Color Display of Images}

This section is tailored for images acquired using an FDA approved and commercially available subwoofer-based system for generating shear waves (Resoundant, MN, USA). For images generated using other in-house developed hardware and software, image display should be optimized as recommended by the manufacturer.

For image viewing purposes, the following information should preferably be generated (Fig. 7).

1. Unwrapped wave images clearly depicting the propagation of shear waves through the abdomen. Phase wrapping occurs when the shear wave motion is large. Since MRE is a phasebased technique, the displacement data typically must be unwrapped before subsequent processing is performed.

2. A gray-scale elastogram without a superimposed $95 \%$ confidence map. 
3.6 Drawing Regions of Interest to Measure Stiffness
3. A gray-scale elastogram with a super imposed $95 \%$ confidence map. The confidence map displayed is a statistical derivation used to overlay a "checkerboard" on the stiffness map to exclude regions that have less reliable (i.e., noisy and discontinuous) stiffness data so that a high-quality and valid stiffness measurement can be obtained.

4. A color elastogram without a superimposed $95 \%$ confidence map. The color elastogram, typically generated in JPEG format is generally used for qualitative stiffness evaluation and visual purposes. However, if the color elastograms are generated in DICOM format, then they can also be used to obtain quantitative measurements.

5. And a color elastogram with a superimposed $95 \%$ confidence map.

The gray-scale elastogram, generated and saved in DICOM format is commonly used to obtain quantitative stiffness measurement, in kiloPascals $(\mathrm{kPa})$. The color elastogram used clinically has a stiffness display range of $0-8 \mathrm{kPa}$. A $0-20 \mathrm{kPa}$ color elastogram display range may also be created and is useful for appreciating tissue stiffness heterogeneity across the larger region. For research purposes, the range may be adjusted as desired.

Mean shear stiffness of the organ is calculated using manually specified regions of interest (ROIs). Skilled interpretation of the magnitude and wave images is currently required to select a ROI from which the average hepatic stiffness can be calculated from the elastogram. Areas with wave interference and noise, as well as partial volume effects and hepatic blood vessels, can bias the stiffness calculation and need to be avoided when selecting this ROI. The ROIs are drawn manually in the largest possible area of organ parenchyma in which coherent shear waves are visible. To avoid areas of incoherent waves, avoid regions immediately under the paddle and stay $\sim 1 \mathrm{~cm}$ inside the organ boundary and contain a minimum of 500 pixels per slice. ROIs should be placed in individual slices. The ROI should also exclude areas of low confidence, as seen by the checkerboard pattern in the masked elastogram images. The procedure needs to be repeated for every slice, with magnitude, phase, and elasticity images all needing to be analyzed. The confidence maps calculated during the stiffness inversion as the fit of a smooth polynomial to the phase images may be helpful in guiding the ROIs across multiple slices. The mean value is calculated from all slices and reported as stiffness in $\mathrm{kPa}$. In practice, the ROIs may be drawn in a single step, keeping these principles in mind. If available, an automated tool capable of deriving ROIs from MRE images and accurately calculating stiffness may be helpful to reduce measurement variability $[32,33]$. 


\subsection{Image Interpretation}

\subsection{Role \\ of Preclinical MRE}

\subsection{Application to Renal Disease Models}

Overall mean stiffness of organ is reported by recording the mean stiffness value of each ROI and then calculating the mean value, weighted by ROI size.

Example: Slice 1: mean stiffness $=2.42 \mathrm{kPa}$ and $\mathrm{ROI}$ size $=$ $2000 \mathrm{~mm}^{2}$; Slice $2:$ mean stiffness $=2.55 \mathrm{kPa}$ and ROI size $=$ $1800 \mathrm{~mm}^{2}$; Slice 3 : mean stiffness $=2.92 \mathrm{kPa}$ and ROI size $=$ $800 \mathrm{~mm}^{2}$; and Slice 4: mean stiffness $=4.22 \mathrm{kPa}$ and ROI size $=1000 \mathrm{~mm}^{2}$; then the weighted mean $=((2.42 \times 2000)+$ $(2.55 \times 1800)+(2.92 \times 800)+(4.22 \times 1000)) /(2000+1800+$ $800+1000)=2.85 \mathrm{kPa}$.

Since MRE is a relatively new, multistep MR technique, there is room for improvement of efficacy, expansion and exploration. There is evidently a need for preclinical applications of the method, primarily to validate the technique against invasive measures and better understand changes in different diseases. Preclinical work helps to realize some of this potential with the exploration of novel driving and contrast mechanisms to improve the efficiency of MRE and to test and provide clinical capabilities that are otherwise unavailable.

Acute kidney injury (AKI) is a sudden episode of kidney failure or kidney damage that happens within a few hours or a few days of an insult such as administration of nephrotoxins. AKI is common in patients who are hospitalized, have underlying kidney disease and especially in older adults. Medullary hypoxic injury plays a major role in the pathogenesis of AKI [34], as has been studied by MRE.

A typical hardware setup for pig MRE is shown in Fig. 8. In a study by Warner et al., the authors tested the hypothesis that renal blood flow (RBF) is an important determinant of renal stiffness as measured by MRE [35]. In this study, in six anesthetized pigs with renal artery stenosis, MRE studies were performed to determine cortical and medullary elasticity during acute graded decreases in RBF (by $20 \%, 40 \%, 60 \%, 80 \%$, and $100 \%$ of baseline) achieved by a vascular occlusion (Figs. 9 and 10). Three sham-operated swine, that served as control pigs, were anesthetized (telazol $5 \mathrm{mg} / \mathrm{kg}$ and xylazine $2 \mathrm{mg} / \mathrm{kg}$ ) and maintained with mechanical ventilation of $1-2 \%$ isoflurane in room air. An ear vein catheter was introduced for saline infusions $(5 \mathrm{~mL} / \mathrm{min})$. MRE studies were performed on a standard clinical 3.0 T MRI system (GE Healthcare, Waukesha, WI) using a standard multichannel phased array coil. After localization, a renal volume series was performed in the axial plane with a T2 weighted fast spin echo sequence using the following parameters: $\mathrm{TR} / \mathrm{TE}=2000 \mathrm{~ms} / 88 \mathrm{~ms} ;$ Flip angle $=90 ; \mathrm{FOV}=35 \mathrm{~cm}$; imaging matrix $=384 \times 320 \times 40$; thickness $=2.5 \mathrm{~mm} ; \mathrm{NEX}=1$. The MRE acquisition sequence used was SE-EPI method. Shear waves were induced by two cylindrical passive pneumatic drivers aligned along the posterior body wall. Continuous vibrations at a 


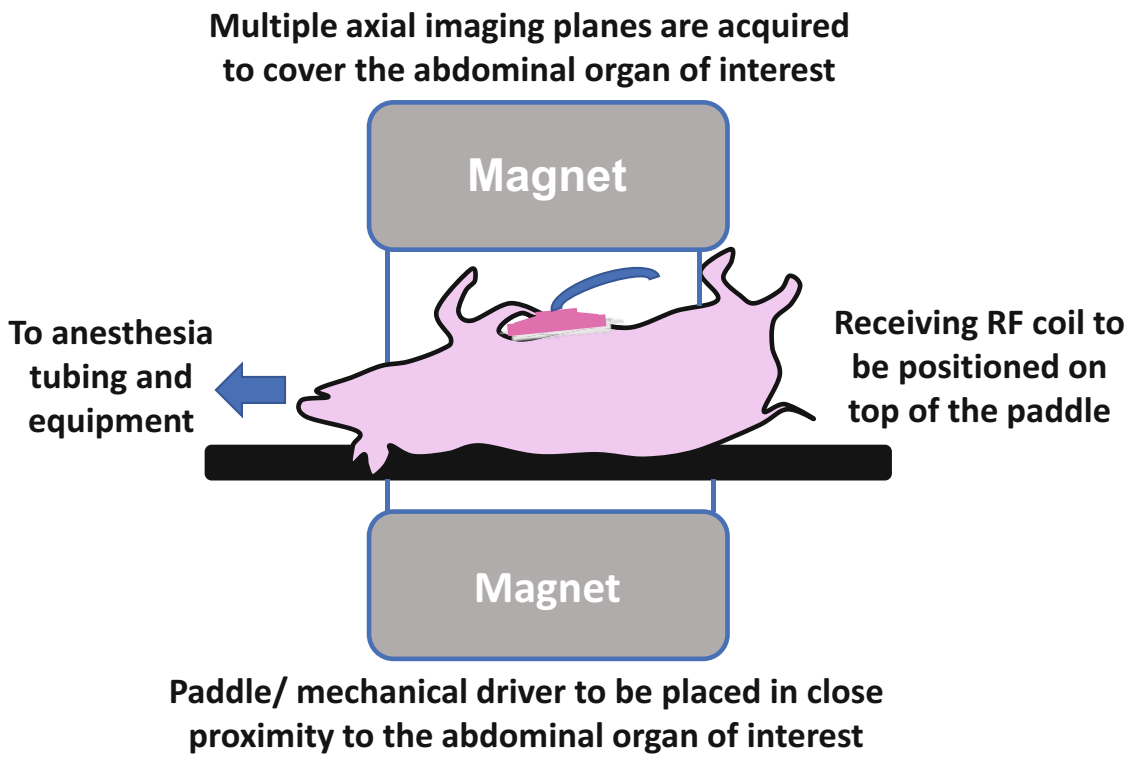

Fig. 8 A typical experimental setup for a pig MRE study
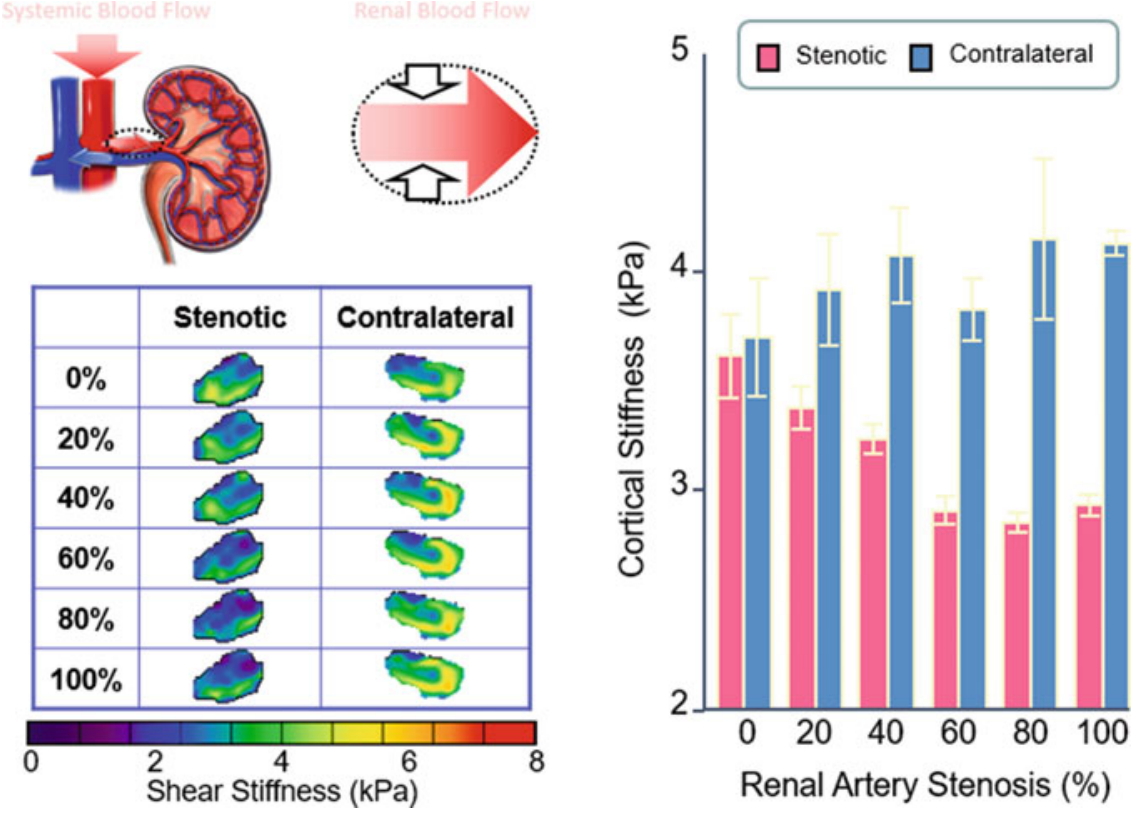

Fig. 9 A representative example from one of the pigs' kidney MRE is shown. The stenosis extent changed gradually from $0 \%$ to $100 \%$. Meanwhile, the systemic blood pressure also rose during the acute decrements in renal blood flow from $75 \pm 3$ to $96 \pm 3 \mathrm{mmHg}$. The kidney's elastograms at six different extents of acute stenosis are shown. The chart summarizes the mean kidney stiffness measurements in stenotic and contralateral sides respectively. From the left elastograms and the right chart, the authors observed that the renal cortex stiffness decreased progressively as the degree of stenosis increased. It is another evidence that less perfusion causes reduced stiffness. Conversely, the contralateral kidney slightly increased in the stiffness during the same period. It is caused by increased systemic blood pressure 


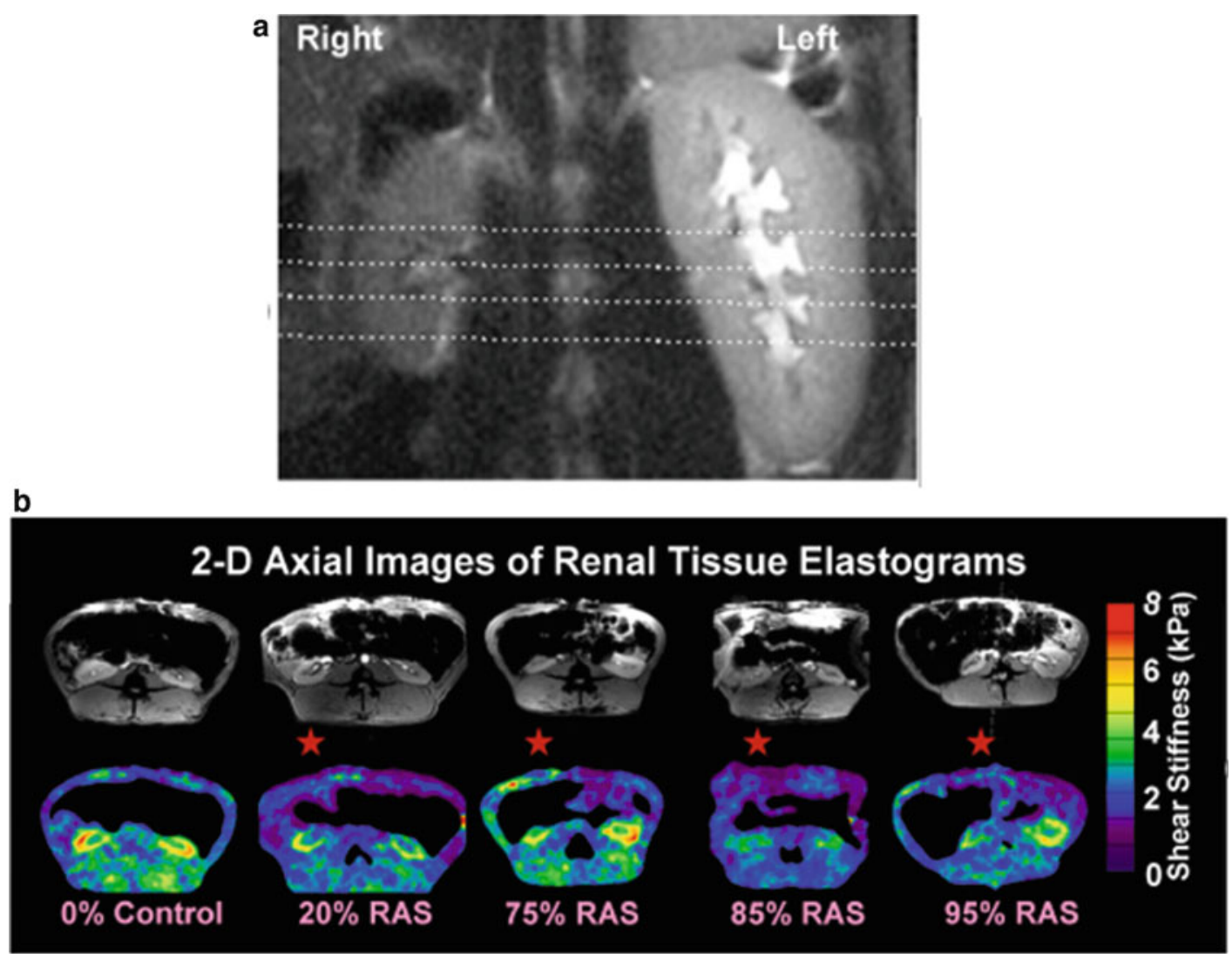

Fig. 10 (a) Right (stenotic) and left (contralateral) kidneys in experimental renal artery stenosis. (b) 2D axial magnitude and elastograms for normal (control) and variable renal artery stenosis at 10 weeks. The elastogram shear stiffness legend is on the far right. Red stars indicate stenotic kidneys. The images were oriented to show the stenotic kidney on the left

motion encoding gradient (MEG) frequency of $120 \mathrm{~Hz}$ generated shear waves throughout the abdominal tissues. MRI parameters used were: $\mathrm{TR} / \mathrm{TE}=2334 \mathrm{~ms} / 48 \mathrm{~ms} ; \mathrm{FOV}=35 \times 35 \mathrm{~cm}^{2}$; Imaging Matrix $=96 \times 96$; Slice thickness $=3 \mathrm{~mm} ; \mathrm{NEX}=1$; Number of shots $=2$; parallel imaging ASSET factor $=2$; four phase offsets through one cycle of motion, six pairs of tetrahedral trapezoidal motion encoding gradients in three orthogonal directions with one motion encoding gradient on each side of the refocusing pulse synchronized with motion. The main finding of their study was that acute decreases in renal blood flow with no fibrosis lead to a decrease in renal cortical shear stiffness. Similarly, in chronic renal arterial stenosis the decrease in renal blood flow offsets a likely increase in stiffness secondary to development of renal fibrosis. Decreased renal stiffness may therefore mask the presence of renal fibrosis, but nevertheless stiffness is a marker of renal hypoperfusion. 


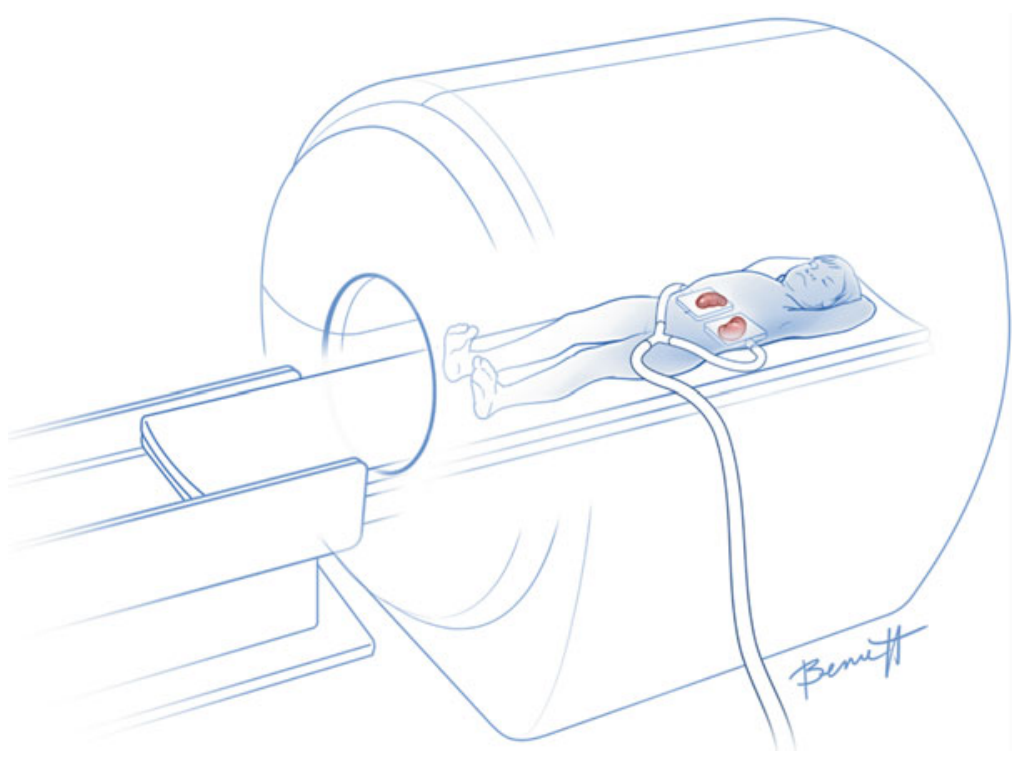

Fig. 11 MRE paddle placement on dorsal side
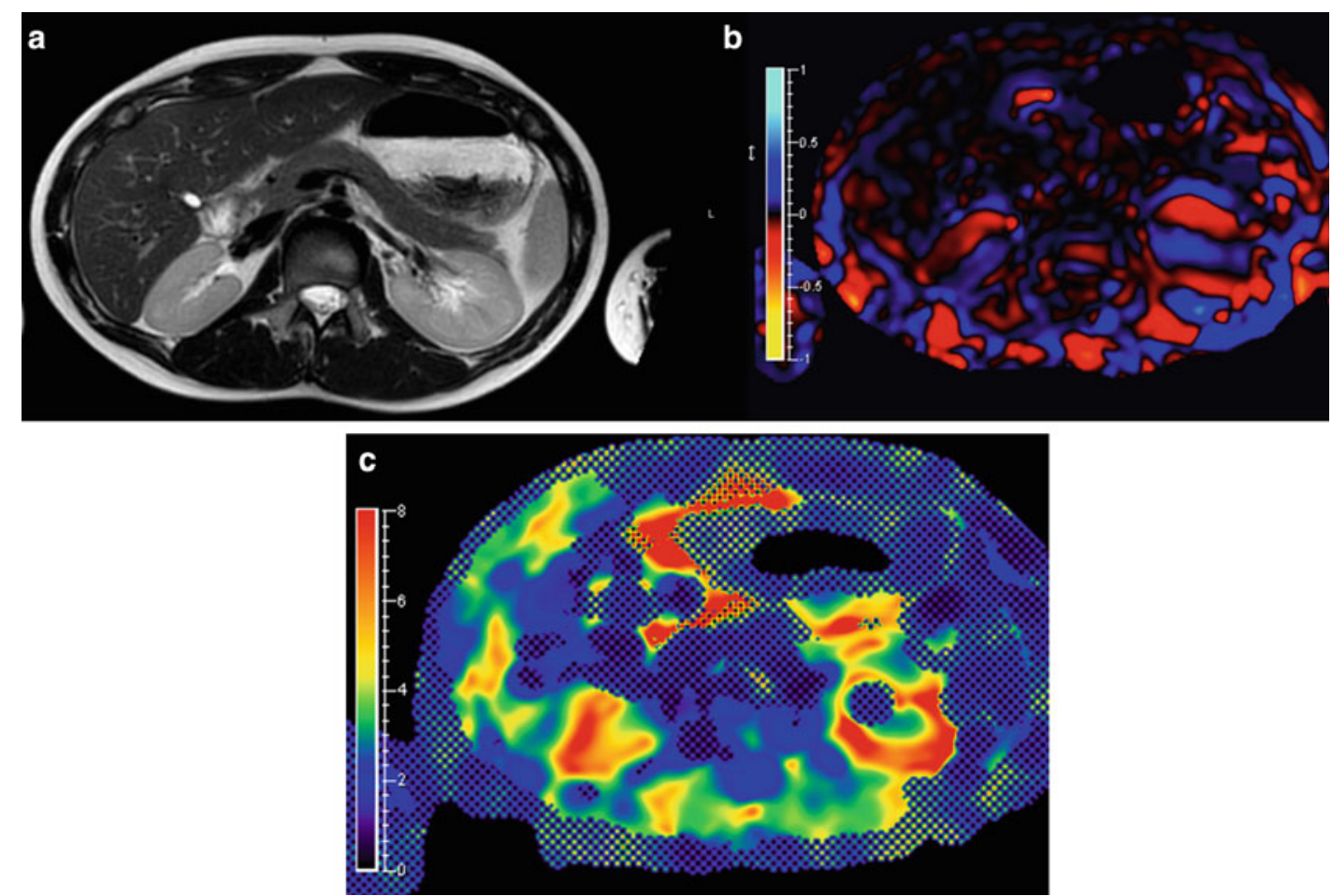

Fig. 12 MRE of the kidneys in a normal 15-year-old male volunteer. (a) T2W images in axial plane. (b) Wave illumination and (c) corresponding stiffness map. Note good illumination of waves in both kidneys (Image courtesy of Dr. Jonathan R. Dillman, Cincinnati, Ohio, USA) 

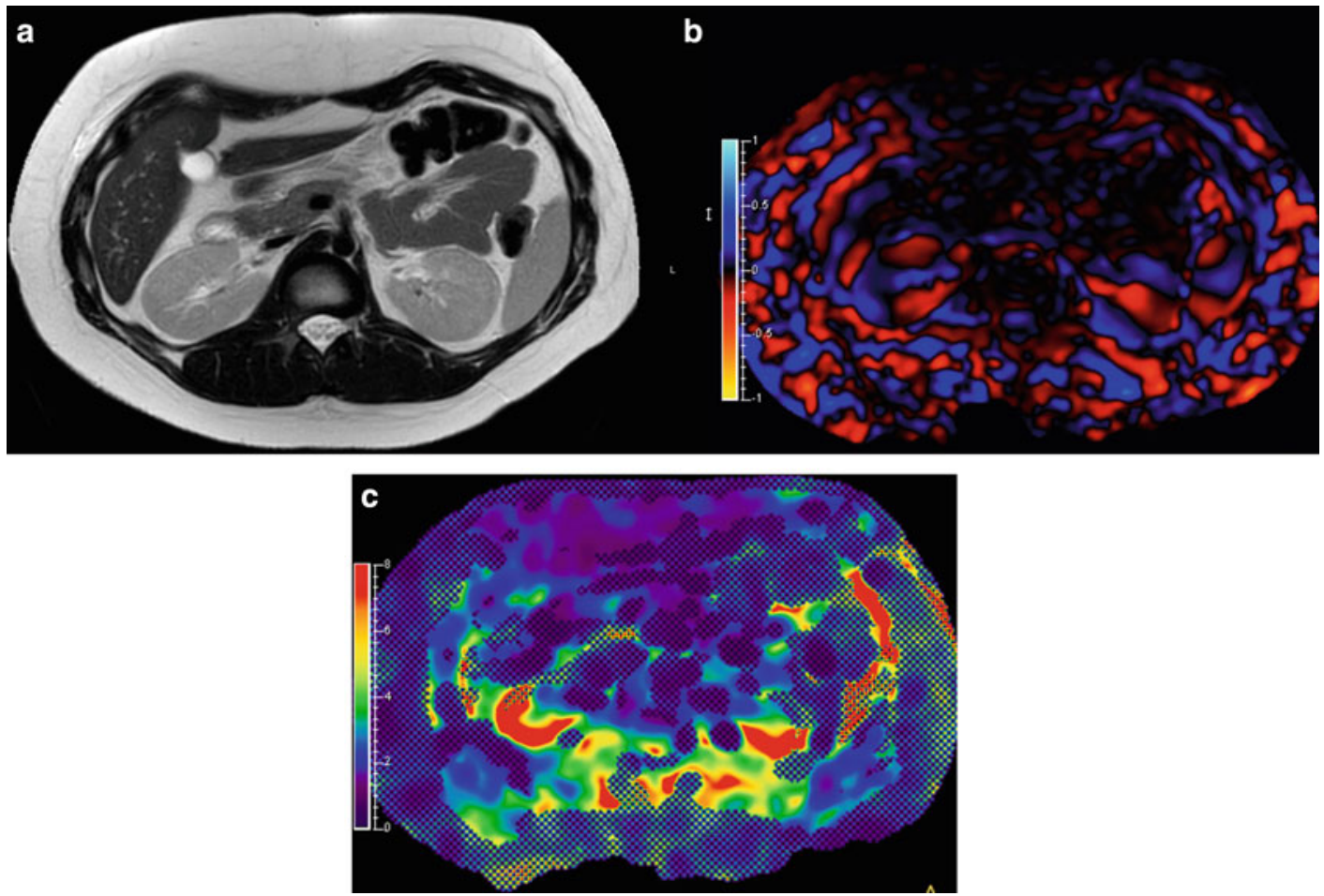

Fig. 13 MRE of the kidneys of a 13-year-old female patient with loss of corticomedullary differentiation; chronic kidney disease. (a) T2W images in axial plane. (b) Wave illumination and (c) corresponding stiffness map (Image courtesy of Dr. Jonathan R. Dillman, Cincinnati, Ohio, USA)
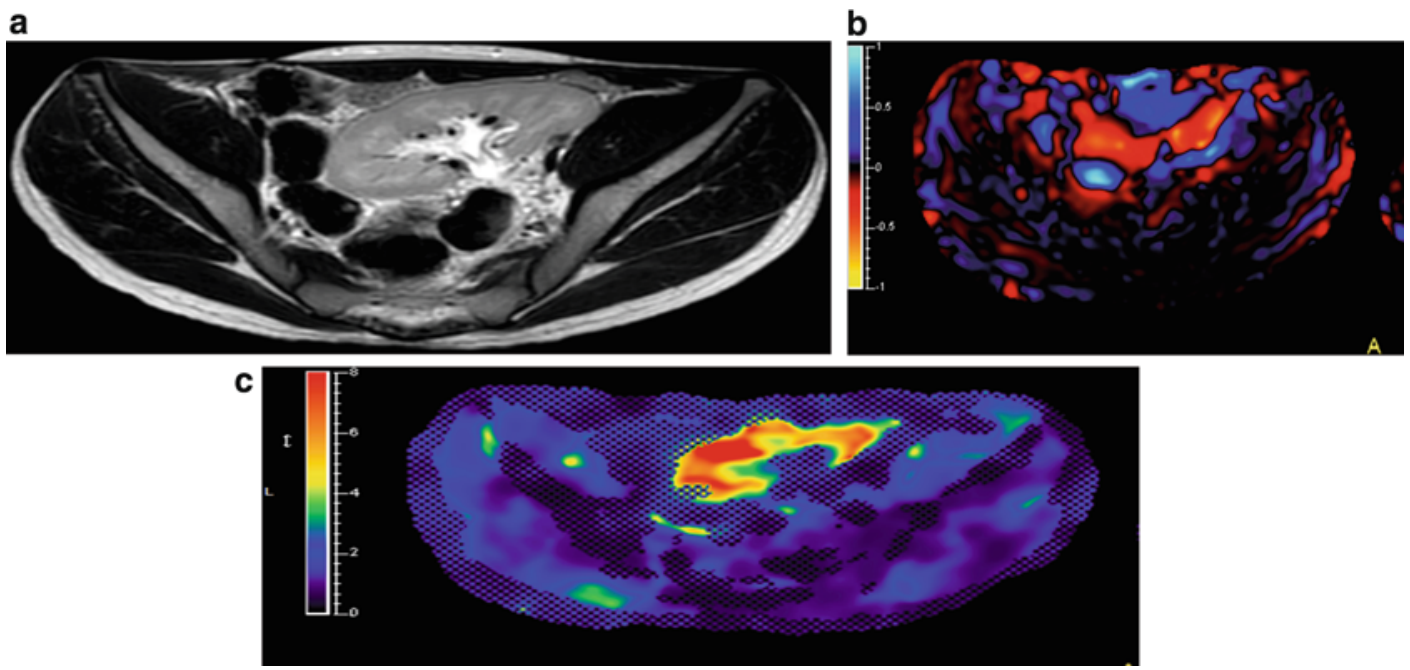

Fig. 14 MRE of the kidneys in a 20-year-old patient with transplant rejection. (a) T2W images in axial plane. (b) Wave illumination and (c) corresponding stiffness map (Image courtesy of Dr. Jonathan R. Dillman, Cincinnati, Ohio, USA) 
Renal MRE has also been demonstrated to provide clinical utility in humans. In clinical renal MRE, the paddle is recommended to be placed on the dorsal side with the patient lying in supine position (Fig. 11). Preliminary results show successful transmission of waves by placing the driver in this position (Figs. 12, 13, and 14). In a recent study by Kirpalani et al., the authors examined whether MRE could noninvasively estimate allograft fibrosis and predict progression of allograft dysfunction [36]. In this study, renal MRE was performed on a standard clinical 3.0 T MRI scanner (Skyra, Siemens Healthineers, USA). Patients were positioned head first and supine, and they were imaged with a standard 18-channel torso phased array coil centered over the allograft. The mechanical vibrations required for MRE were supplied by an active pneumatic driver system. MRE acquisitions were performed using a MEG of $60 \mathrm{~Hz}$ vibrations and a free-breathing, flow-compensated two-dimensional gradient echo coronal MRE pulse sequence to derive magnitude images and a corresponding stiffness map. The imaging orientation was standardized to the coronal plane, with the frequency encoding direction superior to inferior. Other imaging parameters were as follows: field of view, $32 \times 40 \mathrm{~cm}^{2}$; acquisition matrix, $128 \times 128$ reconstructed to $1.48 \times 1.48 \mathrm{~mm}^{2}$ in-plane resolution; no parallel imaging; five two-dimensional slices through the allograft, with each slice being 5 -mm thick with a 1 -mm interslice gap; TR/TE $=50 \mathrm{~ms} / 21 \mathrm{~ms}$. Four time points of the motion and three mutually perpendicular directions of the vector motion were sampled. The acquisition time per scan was reported to be approximately $5 \mathrm{~min}$. This study is based on the hypothesis that fibrosis stiffens organs as soft healthy tissue is replaced by stiff extracellular matrix and suggests the potential of renal MRE as a new noninvasive way to assess whole-allograft fibrosis burden; one that may predict future changes in kidney function.

Renal fibrosis causes an increase in the interstitial extracellular matrix and reduction in the number of tubules, thereby making the kidneys stiffer. Previous studies suggest that renal fibrosis is a distinctive feature of progressive kidney diseases such as chronic kidney disease (CKD), a change that increases the risk of cardiovascular diseases. Therefore, renal fibrosis is an important hallmark for diagnosing CKD. Noninvasive MRE is attractive for clinical use in humans.

Existing imaging technologies have been very successful in the noninvasive assessment of many focal diseases of the kidneys, but they have been somewhat less helpful for evaluating diffuse (medical) renal disease such as glomerulonephritis, and renal biopsy remains the diagnostic tool of choice in many situations. It is now feasible to quantitatively image the shear stiffness of both kidneys with an MRE employing a large, posteriorly located passive acoustic driver. Wave images and the corresponding elastograms can be relatively easily acquired in axial, coronal, and sagittal planes. 
Preliminary results provide a basis and motivation for investigating the potential to use MRE as a diagnostic tool for characterizing renal parenchyma in diffuse and focal diseases of the kidneys.

\section{References}

1. Muthupillai R, Ehman RL (1996) Magnetic resonance elastography. Nat Med 2 (5):601-603

2. Yin M, Talwalkar JA, Glaser KJ, Manduca A, Grimm RC, Rossman PJ, Fidler JL, Ehman RL (2007) Assessment of hepatic fibrosis with magnetic resonance elastography. Clin Gastroenterol Hepatol 5(10):1207-1213.e1202. https://doi.org/10.1016/j.cgh.2007.06.012

3. Yin M, Woollard J, Wang X, Torres VE, Harris PC, Ward CJ, Glaser KJ, Manduca A, Ehman RL (2007) Quantitative assessment of hepatic fibrosis in an animal model with magnetic resonance elastography. Magn Reson Med 58 (2):346-353. https://doi.org/10.1002/ mrm. 21286

4. Xanthakos SA, Podberesky DJ, Serai SD, Miles L, King EC, Balistreri WF, Kohli R (2014) Use of magnetic resonance elastography to assess hepatic fibrosis in children with chronic liver disease. J Pediatr 164 (1):186-188. https://doi.org/10.1016/j. jpeds.2013.07.050

5. Klinkhammer BM, Goldschmeding R, Floege J, Boor P (2017) Treatment of renal fibrosis-turning challenges into opportunities. Adv Chronic Kidney Dis 24 (2):117-129. https://doi.org/10.1053/j. ackd.2016.11.002

6. El Nahas M (2005) The global challenge of chronic kidney disease. Kidney Int 68 (6):2918-2929. https://doi.org/10.1111/j. 1523-1755.2005.00774.x

7. Venkatesh SK, Ehman RL (2014) Magnetic resonance elastography of liver. Magn Reson Imaging Clin N Am 22(3):433-446. https:// doi.org/10.1016/j.mric.2014.05.001

8. Serai SD, Towbin AJ, Podberesky DJ (2012) Pediatric liver MR elastography. Dig Dis Sci 57 (10):2713-2719. https://doi.org/10.1007/ s10620-012-2196-2

9. Venkatesh SK, Ehman RL (2015) Magnetic resonance elastography of abdomen. Abdom Imaging 40(4):745-759. https://doi.org/10. 1007/s00261-014-0315-6

10. Serai SD, Obuchowski NA, Venkatesh SK, Sirlin $\mathrm{CB}$, Miller FH, Ashton E, Cole PE, Ehman RL (2017) Repeatability of MR elastography of liver: a meta-analysis. Radiology 285
(1):92-100. https://doi.org/10.1148/radiol. 2017161398

11. Trout AT, Serai S, Mahley AD, Wang H, Zhang Y, Zhang B, Dillman JR (2016) Liver stiffness measurements with MR elastography: agreement and repeatability across imaging systems, field strengths, and pulse sequences. Radiology 281(3):793-804. https://doi.org/ $10.1148 /$ radiol.2016160209

12. Othman SF, Curtis ET, Plautz SA, Pannier AK, Butler SD, Xu H (2012) MR elastography monitoring of tissue-engineered constructs. NMR Biomed 25(3):452-463. https://doi. org $/ 10.1002 / \mathrm{nbm} .1663$

13. Serai SD, Dillman JR, Trout AT (2017) Spinecho echo-planar imaging MR elastography versus gradient-echo MR elastography for assessment of liver stiffness in children and young adults suspected of having liver disease. Radiology 282(3):761-770. https://doi.org/ $10.1148 /$ radiol.2016160589

14. Schuppan D, Ashfaq-Khan M, Yang AT, Kim YO (2018) Liver fibrosis: direct antifibrotic agents and targeted therapies. Matrix Biol 68-69:435-451. https://doi.org/10.1016/j. matbio.2018.04.006

15. Alukal JJ, Thuluvath PJ (2019) Reversal of NASH fibrosis with pharmacotherapy. Hepatol Int 13(5):534-545. https://doi.org/10. 1007/s12072-019-09970-3

16. Ismail MH, Pinzani M (2009) Reversal of liver fibrosis. Saudi J Gastroenterol 15(1):72-79. https://doi.org/10.4103/1319-3767.45072

17. Lee SY, Kim SI, Choi ME (2015) Therapeutic targets for treating fibrotic kidney diseases. Transl Res 165(4):512-530. https://doi.org/ $10.1016 / \mathrm{j}$.trsl.2014.07.010

18. Tampe D, Zeisberg M (2014) Potential approaches to reverse or repair renal fibrosis. Nat Rev Nephrol 10(4):226-237. https://doi. org/10.1038/nrneph.2014.14

19. Bledsoe G, Shen B, Yao Y, Zhang JJ, Chao L, Chao J (2006) Reversal of renal fibrosis, inflammation, and glomerular hypertrophy by kallikrein gene delivery. Hum Gene Ther 17 (5):545-555. https://doi.org/10.1089/hum. 2006.17.545

20. Braun J, Braun K, Sack I (2003) Electromagnetic actuator for generating variably oriented shear waves in MR elastography. Magn Reson 
Med 50(1):220-222. https://doi.org/10. $1002 / \mathrm{mrm} .10479$

21. Chan QC, Li G, Ehman RL, Grimm RC, Li R, Yang ES (2006) Needle shear wave driver for magnetic resonance elastography. Magn Reson Med 55(5):1175-1179. https://doi.org/10. $1002 / \mathrm{mrm} .20856$

22. Tse ZT, Chan YJ, Janssen H, Hamed A, Young I, Lamperth M (2011) Piezoelectric actuator design for MR elastography: implementation and vibration issues. Int $\mathrm{J}$ Med Robot Comput Assist Surg 7(3):353-360. https://doi.org/10.1002/rcs.405

23. Mansfield P (1977) Multi-planar image formation using NMR spin echoes. J Phys C Solid State Phys 10(3):L55. https://doi.org/10. 1088/0022-3719/10/3/004

24. MAUDSLEY PMAA (1977) Planar spin imaging by NMR. J Magn Reson Imaging 27:101-119

25. Calle-Toro JS, Serai SD, Hartung EA, Goldberg DJ, Bolster BD Jr, Darge K, Anupindi SA (2019) Magnetic resonance elastography SE-EPI vs GRE sequences at $3 \mathrm{~T}$ in a pediatric population with liver disease. Abdom Radiol. https://doi.org/10.1007/s00261-018-1884-6

26. Gandhi D, Kalra P, Raterman B, Mo X, Dong H, Kolipaka A (2019) Magnetic resonance elastography of kidneys: SE-EPI MRE reproducibility and its comparison to GRE MRE. NMR Biomed 32(11):e4141. https:// doi.org/10.1002/nbm.4141

27. Doyley MM (2012) Model-based elastography: a survey of approaches to the inverse elasticity problem. Phys Med Biol 57(3): R35-R73. https://doi.org/10.1088/0031$9155 / 57 / 3 / \mathrm{r} 35$

28. Oliphant TE, Manduca A, Ehman RL, Greenleaf JF (2001) Complex-valued stiffness reconstruction for magnetic resonance elastography by algebraic inversion of the differential equation. Magn Reson Med 45(2):299-310. https://doi.org/10.1002/1522-2594( 200102)45:2<299::aid-mrml039>3.0.co;2-o

29. Manduca A, Oliphant TE, Dresner MA, Mahowald JL, Kruse SA, Amromin E, Felmlee JP, Greenleaf JF, Ehman RL (2001) Magnetic resonance elastography: non-invasive mapping of tissue elasticity. Med Image Anal 5
(4):237-254. https://doi.org/10.1016/ s1361-8415(00)00039-6

30. Low G, Owen NE, Joubert I, Patterson AJ, Graves MJ, Glaser KJ, Alexander GJ, Lomas DJ (2015) Reliability of magnetic resonance elastography using multislice two-dimensional spin-echo echo-planar imaging (SE-EPI) and three-dimensional inversion reconstruction for assessing renal stiffness. J Magn Reson Imaging 42(3):844-850. https://doi.org/10. $1002 /$ jmri. 24826

31. Gandhi D, Kalra P, Raterman B, Mo X, Dong H, Kolipaka A (2019) Magnetic resonance elastography-derived stiffness of the kidneys and its correlation with water perfusion. NMR Biomed 33(4):e4237. https://doi. org $/ 10.1002 / \mathrm{nbm} .4237$

32. Dzyubak B, Venkatesh SK, Glaser K, Yin M, Talwalkar J, Chen J, Manduca A, Ehman RL (2013) Stable automated segmentation of liver MR elastography images for clinical stiffness measurement. Proceedings of SPIE-the international society for optical engineering 8672 . https://doi.org/10.1117/12.2006943

33. Dzyubak B, Venkatesh SK, Manduca A, Glaser KJ, Ehman RL (2016) Automated liver elasticity calculation for MR elastography. J Magn Reson Imaging 43(5):1055-1063. https:// doi.org/10.1002/jmri.25072

34. Hultstrom M, Becirovic-Agic M, Jonsson S (2018) Comparison of acute kidney injury of different etiology reveals in-common mechanisms of tissue damage. Physiol Genomics 50 (3):127-14l. https://doi.org/10.1152/ physiolgenomics.00037.2017

35. Warner L, Yin M, Glaser KJ, Woollard JA, Carrascal CA, Korsmo MJ, Crane JA, Ehman RL, Lerman LO (2011) Noninvasive in vivo assessment of renal tissue elasticity during graded renal ischemia using MR elastography. Investig Radiol 46(8):509-514. https://doi.org/10. 1097 /RLI.0b013e3182183a95

36. Kirpalani A, Hashim E, Leung G, Kim JK, Krizova A, Jothy S, Deeb M, Jiang NN, Glick L, Mnatzakanian G, Yuen DA (2017) Magnetic resonance elastography to assess fibrosis in kidney allografts. Clin J Am Soc Nephrol 12(10):1671-1679. https://doi. org/10.2215/cjn.01830217

Open Access This chapter is licensed under the terms of the Creative Commons Attribution 4.0 International License (http://creativecommons.org/licenses/by/4.0/), which permits use, sharing, adaptation, distribution and reproduction in any medium or format, as long as you give appropriate credit to the original author(s) and the source, provide a link to the Creative Commons license and indicate if changes were made.

The images or other third party material in this chapter are included in the chapter's Creative Commons license, unless indicated otherwise in a credit line to the material. If material is not included in the chapter's Creative Commons license and your intended use is not permitted by statutory regulation or exceeds the permitted use, you will need to obtain permission directly from the copyright holder. 